\title{
Sexual Reproduction in Bdelloid Rotifers
}

Veronika N. Laine ${ }^{\mathrm{a}}$, Timothy Sackton ${ }^{\mathrm{b}}$ and Matthew Meselson ${ }^{\mathrm{ct}}$

${ }^{a}$ Current address, Department of Animal Ecology, Netherlands Institute of Ecology, 6708 PB Wageningen, The Netherlands; ' Informatics Group, Faculty of Arts and

Sciences, Harvard University, Cambridge, MA 02138; 'Department of Molecular and Cellular Biology, Harvard University, Cambridge, MA 02138. ${ }^{\dagger}$ Corresponding author: Email: msm@wjh.harvard.edu

CLASSIFICATION: Biological Sciences, Evolution

KEYWORDS: allele sharing, conversion, desiccation, epistasis, horizontal gene transfer, life history, loss of heterozygosity, outcrossing, population structure, Red Queen 


\section{ABSTRACT}

Nearly all eukaryotes reproduce sexually, either constitutively or facultatively, and nearly all that are thought to be asexual arose recently from sexuals, suggesting that loss of sex leads to early extinction. In apparent exception, there are several groups of ancient origin that have been thought to be entirely asexual. Of these, the most extensively studied are the rotifers of Class Bdelloidea. Yet the evidence for their asexuality is entirely negative -- the failure to establish the existence of males or hermaphrodites. Nevertheless, there is a growing body of evidence that bdelloids do reproduce sexually, albeit rarely, retaining meiosisassociated genes and, in a limited study of allele sharing in the bdelloid Macrotrachela quadricornifera, displaying a pattern of genetic exchange indicating recent sexual reproduction. Here we present a much larger study of allele sharing in the same system, clearly showing the occurrence of sexual reproduction, thereby removing the principal challenge to the generalization that sexual reproduction is essential for long-term evolutionary success in eukaryotes. We also discuss the relation between bdelloid life history and population structure and a possible benefit of outcrossing in restoring beneficial genome-wide epistatic interactions disrupted by loss of heterozygosity.

STATEMENT OF SIGNIFICANCE: Many hypotheses have been advanced to explain why, despite its substantial costs, sexual reproduction is nearly universal in eukaryotes and why the loss of sex generally leads to early extinction--a major problem in current evolution theory. Posing a challenge to all such hypotheses are a few groups of ancient origin that have been thought to be entirely asexual. Of these, the most extensively studied are the rotifers of Class Bdelloidea. Here we show that a bdelloid species is facultatively sexual, removing what had been a long-standing challenge to hypotheses for the benefit of sex. We also suggest that genome-wide beneficial epistasis may contribute to the advantage of sex over asex in diploids and to the predominance of diploidy over haploidy in eukaryotes. 
MANY hypotheses have been advanced to explain why, despite its substantial costs, sexual reproduction, meiotic segregation and outcrossing, is nearly universal in eukaryotes and why its loss generally leads to early extinction--a major problem in evolution theory (1-5). Posing a challenge to all such hypotheses are a few groups of ancient origin that have been considered to be entirely asexual (6). Of these, the most extensively studied are the rotifers of Class Bdelloidea (7) (Fig. 1). First described nearly 350 years ago $(8,9)$, bdelloids are minute freshwater invertebrates commonly found in lakes, ponds and streams, swimming free or attached to surfaces, and in ephemerally aquatic habitats such as temporary pools and the water films on lichens and mosses. Characterized by their ciliated head and bilateral ovaries, bdelloids are classified in 4 families, 19 genera and some 500 morphospecies. The bdelloid radiation began tens of millions of years ago, as shown by the synonymous site difference between families and by the presence of bdelloid remains in ancient amber. Although typically only several tenths of a millimeter in size and containing only ca. 1,000 nuclei, mostly in syncytial tissues, they have ganglia, muscles, digestive, excretory, reproductive and secretory systems; photosensitive and tactile sensory organs; and structures for crawling, feeding and swimming. Bdelloids are degenerate tetraploids, descended from an ancient tetraploid ancestor (10-13).

The only observed means of bdelloid reproduction is from eggs produced in welldifferentiated ovaries, with no reduction in chromosome number $(14,15)$. A few days after deposition a young bdelloid emerges and a few days later commences egg-laying, producing up to 32 eggs over a period of up to a few weeks during which there is little death, after which the death rate increases more or less exponentially $(16,17)$. Depending on species and conditions, the mean time from egg deposition to death is about a month (17-19). Bdelloids are eutelic, with no cell division after eclosion except in the germ line.

Bdelloids as a class are extremophiles, being able to survive prolonged desiccation, some species more than others, as well as starvation and extremes of temperature, and to resume reproduction upon restoration of favorable conditions, highly unusual abilities probably apomorphic to the Class (20-22). Bdelloids have an extremely effective system of anti-oxidant protection, as manifested by their extreme 
resistance to ionizing radiation and to IR-induced protein carbonylation $(23,24)$, apparently an adaptation to avoid the oxidative damage caused by desiccation in other systems $(25,26)$.

Although bdelloids have been systematically studied ever since adequate microscopes became available $(27,28)$, there is no confirmed evidence of males or hermaphrodites. It has been estimated that some 500,000 bdelloids from a variety of habitats and from laboratory culture have been examined by contemporary rotifer workers without ever having seen males or hermaphrodites (29). The only report to the contrary, in a treatise otherwise devoted to observations of males and sexual periods of the facultatively sexual rotifers of Class Monogononta, is a hesitant account of having twice seen a single male among many bdelloids of species Rotaria rotatoria "present in almost incredible numbers" under the ice of a frozen lake in Denmark during a population bloom in November 1923, there being almost no bdelloids there the following summer (30). Sampling conducted there in January 2019 found few bdelloids, no males and no ice, the winter of that year having been among the warmest on record (Martin Sorensen, personal communication).

Despite the failure to document the existence of males, it may be that bdelloids reproduce sexually only rarely and under conditions not yet adequately investigated-a possibility made less implausible by estimates that outcrossing of Saccharomyces cerevisiae in the field may occur as seldom as once in 25-50,000 generations $(31,32)$, owing to the repression of meiosis which, however, can be relieved in the laboratory by growth in specific media.

In the following, we review findings once interpreted as evidence for bdelloid asexuality but now known to have other explanations and summarize recent genomic evidence strongly suggestive of bdelloid sex. We then present an extensive study of allele sharing in the bdelloid Macrotrachella quadricornifera demonstrating the occurrence of sexual reproduction. We also discuss a possible benefit of outcrossing in restoring beneficial genome-wide epistatic interactions disrupted by loss of heterozygosity, the relation between bdelloid life history and population structure, and its implications for how bdelloid males and mating might be discovered. 


\section{Findings formerly interpreted as evidence for ancient asexuality}

Heterozygosity. In sexuals, heterozygosity caused by mutation is limited by gametic drift. Supposing that, if anciently asexual, bdelloids might therefore exhibit high levels heterozygosity, Mark Welch and Meselson compared the synonymous site difference between copies of several genes in bdelloids and in facultatively sexual monogonont rotifers. Finding the differences in bdelloids to be much higher than in monogononts and finding the synonymous site mutation rate in bdelloids to be no higher than that in monogononts and considering ancient polyploidy, unknown at the time in any sexuallyreproducing animal, to be unlikely, they interpreted their findings as evidence that bdelloids had evolved asexually $(33,34)$. Continued investigation, however, showed that bdelloids are degenerate tetraploids, and that the highly diverged gene copies are homeologs, not homologs $(10,11)$. Bdelloid heterozygosity, the difference between homologs, lies within the range known for sexuals, providing no evidence for asexuality.

Meanwhile, mutation accumulation studies with asexual Daphnia pulex and Saccharomyces cerevisiae unexpectedly found that the frequency with which a nucleotide site is covered by a tract of homozygosity, as may result from germline mitotic crossing-over at the four-strand stage or by certain processes of DNA damage repair, is much greater than the frequency of nucleotide substitution (35-38). In sexuals, heterozygosity lost by such processes can be regained by outcrossing. But if bdelloids are ancient asexuals and loss of heterozygosity is more frequent than substitution, the lack of outcrossing should be manifested as unusually low heterozygosity, the opposite of what had been thought $(32,39)$. The observation that bdelloid heterozygosity is within the range known for sexual taxa therefore suggests that bdelloids may be sexual, with lost heterozygosity regained by occasional outcrossing. The moderate levels of heterozygosity seen in other putative ancient asexuals $(6,40)$ would then suggest that they too engage in outcrossing.

Paucity of retrotransposons. In a test for asexuality based on the expectation that an asexual population would eventually either become devoid of pre-existing deleterious vertically-transmitted transposable elements or be driven to extinction by their increase $(41,42)$, Arkhipova and Meselson (43-45) surveyed the genomes of bdelloids and of 
monogononts and other sexually-reproducing species of diverse phyla for sequences coding for reverse transcriptases of LINE-like retrotransposons. Such sequences were found to be abundant in all the taxa known to reproduce sexually but were not detected in any of the bdelloids, while DNA transposons, transmitted horizontally, were found to be abundant. Nevertheless, although bdelloids are nearly devoid of LINE-like retrotransposons, later work showed that they are not entirely absent $(46,47)$ and that bdelloids have particularly effective retrotransposon silencing systems (48). Although unusual, the paucity of LINE-like retrotransposons is therefore non-evidentiary as regards bdelloid sexuality. Consistent with asexuality but also not decisive, is the presence of odd chromosome numbers in some bdelloid species $(14,49)$.

Genome structure. Flot et al. (12) reported a draft genome sequence of the bdelloid Adineta vaga having numerous breaks in the colinearity of homologous regions and individual scaffolds containing genes in direct or palindromic repeats but no copy elsewhere in the genome, a situation incompatible with the existence of homologous chromosome pairs and in which segregation with independent assortment would generally give rise to inviable zygotes. These features led the authors to conclude that bdelloids had evolved ameiotically. But subsequent genomic sequencing of three other bdelloid species, including Adineta ricciae, a close relative of $A$. vaga, found that the unusual genomic features that had been interpreted as evidence for ameiotic evolution are largely absent, suggesting that their apparent presence in $A$. vaga resulted from mis-assembly (13) as later shown to be the case by the demonstration of homologous chromosome pairs in this species (50).

\section{Previous Evidence for Sexual Reproduction}

Allele sharing. A finding of two individuals closely related with respect to a given genomic region but more distantly related with respect to its homolog, a form of phylogenetic noncongruence known as allele sharing, would mean that recently in their ancestry the region had undergone some form of genetic exchange between individuals, as by sexual reproduction, homologous horizontal transfer, or parasexuality. Examining the sequences of both homologs in each of four genomic regions 2.4 to 9.7 
$\mathrm{kb}$ in length, Signorovitch et al. (51) found at each region a striking example of allele sharing among three isolates of the bdelloid M. quadricornifera, designated MA, MM, and $\mathrm{CR}$, collected at widely separated sites but all belonging to the same mitochondrial clade (S1 Appendix, Text 1 Methods). At each of the four regions, a homolog of MA was found to be identical to a homolog of MM while the other homolog of MA was identical to a homolog of CR in two regions and nearly so in the other two. All other inter-homolog differences, within and between isolates, almost entirely single nucleotide substitutions, were 1-3 percent, typical of large genetically diverse outbreeding populations. These findings argued strongly for recent sexual exchange but suggested that the four regions may not have recombined, as in certain plants in which entire haplotypes remain intact through meiosis (52). At the time, the suggestion of no recombination appeared to gain support from the earlier although subsequently disproven report that $A$. vaga lacks homologous chromosome pairs and has additional features incompatible with independent assortment and crossing-over (12). The conclusion of Signorovitch et al. $(51,53)$ that the allele sharing they observed was evidence for sexual reproduction was disputed by Debortoli et al. (54) who suggested on the basis of their studies in $A$. vaga that it resulted instead from horizontal transfer. Their evidence for horizontal transfer was challenged, however, by Wilson et al. (55) who showed that it could be explained as the result of cross-contamination among isolates.

Meiosis-related genes. Nowell et al. (13) sequenced the genomes of four bdelloid species belonging to two bdelloid families and searched for the proteomes of 12 genes considered to be meiosis-specific. Proteomes of all but one, red1, were found in all four species but neither was red1 found in Drosophila melanogaster, a sexual species known to lack it. The presence of proteomes of these 11 meiosis-related genes in each of the four bdelloid species suggests that they are under selection for the same functions they serve in sexuals and therefore that bdelloids engage in meiosis. Although Flot et al. (12) did not find five of these genes in their assembly of the A. vaga genome, their detection in four other bdelloid species suggests that they are present in A. vaga as well. 


\section{Homologous chromosome pairs and Hardy-Weinberg Equilibrium}

Recently, phased sequencing of A. vaga and hybridization in situ revealed that its 12 chromosomes constitute 6 homologous pairs, a finding interpreted by the authors as evidence of automixis (50). Other recent sequencing found Hardy-Weinberg equilibrium and a fall-off of linkage with distance within a group of 11 isolates of $A$. vaga, suggested by the authors to be a result of horizontal gene transfer (56). Nevertheless, all these findings may be more conventionally explained as evidence for sexual reproduction with outcrossing and, taken together with the earlier finding of allele sharing in $M$. quadricornifera suggest that bdelloids are facultatively sexual with outcrossing, as clearly shown by the much larger study of allele-sharing reported here.

\section{Results and Discussion}

Alignments. As described more fully in Methods (SI Appendix, S1), we obtained phased sequences of a large number of genomic regions of $M$. quadricornifera isolates MA, MM and CR by aligning individual Nanopore reads of MA against the combined megabubbles of the three isolates obtained by $10 x$ sequencing and assembly with Supernova 2.1.1. From the resulting alignments, we selected those that were co-linear with the Nanopore read and which included a pair of homologs from at least two of the three isolates. The megabubble sequences were then realigned among themselves and trimmed with Gblocks to remove indels, repeats and overhangs, neither adding to nor rearranging sequences. We then rejected the shorter alignments when two or more overlapped and the five alignments in which both homologs of MM or CR differed by more than $10 \%$ from both homologs of MA. This may occur when, owing to a deletion in $\mathrm{MM}$ or $\mathrm{CR}$ occurring since their divergence from MA, there is no MM or CR sequence homologous to the Nanopore read, leaving only the homeologous sequence to align with it. This left for analysis 1,177 non-overlapping alignments representing as many separate genomic regions, 331 with all three isolates, 291 with MA and MM, 110 with MA and CR and 445 with MM and CR, having a mean length of 12,490 bp (range 2,051 - 32,937 bp), altogether covering $14.7 \mathrm{Mb}$, approximately $4 \%$ of the ca. $360 \mathrm{Mb} \mathrm{M}$. quadricornifera genome (SI Appendix, Table S2). Matrices giving pair-wise differences between homologous sequences, phylograms and "tic" plots of the spatial distribution of 
differences between homologs are given in Table 1 for four representative regions and for all 1,177 regions in Supplemental Information (SI Appendix, Tables S1-3).

Frequency of identical sharing. Half of the MA-MM and MA-MM-CR alignments, 315 of 622 , comprise a discrete class in which a homolog of MA is identical to a homolog of MM (Fig. 2B). The frequencies of identical MA-MM sharing in the MA-MM and MA-MMCR alignments considered separately are 0.519 and 0.495 , respectively or 0.506 overall $($ S.E. $=0.02)($ Table 2). MA and MM also share identical homologs with $\mathrm{CR}$, but in a much smaller proportion of the alignments (Table 2; Fig. 2A, C). CR shares identical homologs with MA in 12 of 441 alignments, and with MM in 18 of 776 alignments, or $2.7 \%$ and $2.3 \%$ respectively. In alignments without identical sharing the differences between the homolog of MA most similar to a homolog of MM form a broad distribution with a mean of 0.96 SNPs per 100 bp (S.E. = 0.61), Fig. 2B.

Most or all of the regions identically shared between MA and MM must be considerably longer than the alignments in which we find them, as shown in a plot of the frequency of identical MA-MM sharing against alignment length in consecutive intervals each comprising 76-79 alignments (Fig. 4). The frequency of identical sharing is not significantly different from 50 percent in even the longest alignments.

Genealogy. MA and MM share a homolog in a discrete class amounting to half of the MA-MM and MA-MM-CR alignments while their other homologs, within and between MA and MM, are substantially diverged, as expected for diploids related as grandchild and grandparent, half siblings, aunt and uncle or nephew and niece in a large genetically diverse panmictic population. The near equality of MA-CR and MM-CR sharing frequencies indicates that $\mathrm{CR}$ is equidistant from MA and MM and therefore that MA and $\mathrm{MM}$ are not grandparent and grandchild or aunt/uncle-nephew/niece but rather half siblings or double first cousins.

Inspection of tic plots for the triple alignments reveals a minority with long interior regions of MA-MM identity covering most but not all of the alignment (SI Appendix, Table S5). In these regions there is substantial divergence from CR, showing that such identity is not the result of extreme conservation but instead is likely to reflect more 
remote relationships between $\mathrm{MA}$ and $\mathrm{MM}$ in addition to their relation as half siblings or double first cousins. Similarly, alignments in which CR is identical to MA or MM over much but not all the alignment are likely to reflect remote relationships between CR and MA and between CR and MM. In general, more distant relations will be manifested as shorter regions of identity by descent, owing to the recombination that occurs at each meiosis. For individuals related as half-sibs or double first cousins the regions of identity by descent from their common grandparents, assuming one cross-over per meiosis in each arm of the 10 chromosomes of $M$. quadricornifera, will average several Mbp in length, far longer than our longest alignments, consistent with the observation that the frequency of identically shared regions we see does not fall off with their length (Fig. 4).

Homozygosity. In each of the three isolates there are a few regions that are entirely homozygous (Table 2). No more frequent in the shorter half of the alignments than in the longer half, they must generally be longer than the alignments in which they occur. These regions may be identical by descent or may have arisen by conversion or by germ-line mitotic crossing-over. The few alignments in which these processes may have erased evidence of sharing are not included in the totals given above or in Table 2.

Recombination. The pattern of sharing in each of the four regions examined by Signorovitch et al. (51), in which MA shares homologs with both MM and CR, suggested to them that entire haplotypes may have passed through meiosis without recombination. In contrast, we find this pattern only 4 of our 331 much longer MM-MA-CR alignments. It may be that the pattern encountered in the earlier study resulted from identity by descent from remote ancestors, in which case such regions would generally be shorter than our alignments and therefore not counted as allele sharers. That recombination definitely occurs is seen in the observation that MA and MM do not share entire haplotypes but only a quarter of their genomes and share even less with CR and as is also evident in the many alignments in which there are segments of identical sharing. 


\section{Horizontal transfer, Contamination and Parasexuality}

That 315 of the 622 regions we examined in MA and MM share an identical allele cannot be explained as the result of horizontal transfer, parasexuality or any sort of contamination. For this to have occurred by HGT would require massive transfer of long DNA segments coincidentally at just half the regions and somehow prevented from ever transferring both homologs, the configuration $\mathrm{a} / \mathrm{b} \mathrm{a} / \mathrm{b}$ being absent from the alignments that include MA and MM. The absence of such double sharing and, independently, the remapping of the Illumina reads on the 1,177 haplotypes (SI Appendix Text S1, Methods), show that there was no contamination in the input DNA, the sequencing or anywhere in the workflow leading to our 1,177 alignments.

Neither can the observed allele sharing be explained as the result of parasexuality. In the parasexual cycle, known only in certain fungi and protozoans, nuclei from two individuals fuse, forming nuclei of doubled ploidy that during subsequent generations undergo occasional mis-division, only rarely yielding viable diploids. In bdelloids, this would require nuclei from different individuals, sequestered in ovaries within the body of the animal, somehow to undergo fusion, followed by a series of random chromosome losses to give viable segregants, all having ten chromosomes.

\section{Number of generations since the homologs shared by MA and MA separated. The} number of generations since the MA-MM sharing event may be estimated from the frequency of substitution differences between shared homologs; from the number of generations that would cause mutational reduction of the frequency of identical sharers

to fall significantly below the observed value of 0.5 ; from the frequency of homozygosity; and from the mitochondrial difference between MA and MM.

MA and MM differ by a single substitution in 2 of the 291 MA-MM alignments and in 4 of the 622 MA-MM-CR alignments. For a mean alignment length of $15 \mathrm{~kb}$ this is a frequency of $0.44 \times 10^{-6}$ per bp. Substitution rates measured in accumulation experiments with Caenorhabditis elegans, asexual $D$. pulex, and $D$. melanogaster range from 2.3 to $5.5 \times 10^{-9}$ per generation (37). Taking a substitution rate of $4 \times 10^{-9}$ and assuming a Poisson distribution of the small differences between shared homologs, as 
expected for mutation, this suggests that the shared homologs may be separated by 100-200 generations.

If there were as many as 2,000 generations separating the shared homologs of MA and MM and again assuming a substitution rate of $4 \times 10^{-9}$ per generation, the expected number of substitutions in regions 18-33 kb in length, the longest interval in Figure 4 , would be $0.14-0.26$, reducing the proportion of identical sharers to $0.43-$ 0.39 , substantially less than what we observe, suggesting that the number of generations between the shared homologs is no more than about 1,000.

As tracts of homozygosity arising in a large genetically diverse, essentially panmictic population are generally made heterozygous by outcrossing, the frequency of substitutions in such tracts will increase with the number of generations since the last outcross. Assuming the likelihood of a site being covered by a tract of homozygosity to be $4 \times 10^{-5}$ per generation (35-38) and considering that the total length of MA regions is 13.4 Mbp of which perfectly homozygous regions comprise some $151 \mathrm{kbp}$, or about $1.1 \%$, it would appear that there have been some 340-500 generations from when the shared homologs separated to MA and 550-800 generations to MM.

A fourth estimate of the number of generations since homologs of MA and MM separated may be obtained by assuming that their mitochondria descended from a shared mother or maternal grandmother. Taking the difference of 5 substitutions or 2.5 $X 10^{-5}$ between their $14 \mathrm{~kb}$ mitochondria (57) and a mitochondrial mutation rate of $1.5 \mathrm{X}$ $10^{-7}(37,58)$, would suggest that the shared homologs separated some 170 generations ago. Altogether, these various estimates suggest that the shared homologs are separated by no more than about a thousand generations.

Abundance of close relatives in the sampled population. Isolates MA, MM and CR were collected at widely separated sites as part of a collection of 29 individuals having the morphology of Family Philodinidae (51) (SI Appendix,S1 Methods). What aspects of bdelloid life history could make finding relatives as close as MA and MM in so small and widely dispersed a sample of what must be an enormous population? It must be that the sampled population is largely made up of relatively few, very large, widely dispersed clones descended from recent crossing. 
Such an unusual population structure might result if sexual periods occur only rarely, during a population bloom, with multiple rounds of random mating among two or more founding types and their progeny, producing large numbers of closely related individuals. This, followed by wide dispersion and extensive clonal reproduction would give rise to very large, widely dispersed clones of the products of recent crosses. Meanwhile, lines that fail to outcross would suffer loss of heterozygosity and rapid clonal erosion, as seen in asexual Daphnia pulex (59), driving them to extinction unless revived by timely outcrossing, leaving only widely dispersed descendants from recent crossing in a limited number of blooms.

It may be that males are produced and mating occurs only when particular mating types are present together, each producing a signal that causes the other to produce haploid eggs and haploid males, possibly via mictic females as with monogonont rotifers. At some stage, from fertilization to zygote development, selfing may be prevented and heterozygosity thereby maintained. On this picture, field observations intended to detect males and mating should be made during population blooms, as may require specific external stimuli, and in sizeable bodies of water should it be that different but compatible types must be present in order to initiate mixis. Further, by analogy with monogononts, the appearance of bdelloid males may be confined to only a short interval during a population bloom, therefor requiring frequent sampling for their detection (30).

Bdelloid life history and the benefit of sex. It may be asked if there is a relation between the infrequency of bdelloid outcrossing and bdelloid life history. A distinctive feature of the latter is the ability of bdelloids to withstand desiccation and resume reproduction upon rehydration, an ability not present in most of the fungi and other organisms that infect, parasitize, prey on or compete with bdelloids. In habitats that undergo desiccation and rehydration the population of desiccation-intolerant antagonists will be greatly reduced at each episode of desiccation while bdelloids will resume reproduction upon rehydration. Bdelloids gain additional freedom from having to coevolve with biological antagonists by their ability to survive prolonged starvation, extremes of temperature and exposure to toxic conditions lethal to other taxa $(60,61)$. 
Further, owing to their small mass when desiccated, about 10ug, once airborne, even if associated with a small amount of adhering material, bdelloids may be transported by wind or vectors over considerable distances (62), transferring to an environment where antagonists may be less abundant or less antagonistic. The combination of anhydrobiosis and resistance to conditions inimical to other taxa and dispersibility by wind, birds or other vectors therefore affords substantial protection from what would otherwise be co-evolving biotic antagonists (62-64), reducing the need for frequent recombination by largely eluding the "Red Queen" (65-68).

The ability of bdelloids to survive conditions lethal to co-evolving biotic antagonists and, by becoming dormant, to survive transiently inhospitable environmental conditions without having to produce recombinant progeny in order to maintain the species, raises the question of why bdelloids nevertheless reproduce sexually and what might cause rapid clonal erosion in the absence of sex. In addition to the well-known benefits of outcrossing in covering deleterious recessive mutations, restoring overdominance, and, together with recombination, in purging deleterious mutations and assembling favorable linkage combinations (1-5), an important further benefit of outcrossing may be the restoration of information lost by conversion, mitotic crossing-over or deletion and the consequent disruption of favorable genome-wide epistatic interactions (69-71). Although difficult to study, such genome-wide epistasis is to be expected, maximizing the opportunity for favorable structural interactions between diverse gene products, the so-called "interactome". Such epistasis may also include spatial interactions between chromosome domains, influencing the regulation of gene expression (72). The enhanced opportunity for genome-wide favorable epistasis made possible by diploidy may also contribute to its prevalence over haploidy in eukaryotes generally and to the stability of bdelloid tetraploidy.

\section{Summary}

Our principal finding is that bdelloids are not entirely asexual, removing what has long been a challenge to hypotheses for the evolutionary benefit of sex. Contrary to their reputation as ancient asexuals, bdelloids of the population represented by the isolates of $M$. quadricornifera we have studied and presumably bdelloids as a Class definitely 
engage in occasional sexual reproduction. It is suggested that the infrequency of bdelloid sexual reproduction is an adaptation to the distinctive bdelloid life history. Being able to survive desiccation and other conditions lethal to their biological antagonists, bdelloids are largely relieved of the need to co-evolve with them, therefore requiring only infrequent recombination to maintain fitness. Nevertheless, occasional outcrossing is required in order to allow generation of favorable recombinants and to recover genetic information lost by conversion, mitotic crossing-over or deletion and to cover accumulated deleterious recessive mutations and restore overdominance and beneficial genome-wide epistatic relations. The present evidence for sexual reproduction and outcrossing in a species of bdelloid rotifers, the group once considered the best example of ancient asexuality, makes it likely that there are no ancient asexuals and that sexual reproduction with outcrossing is universally essential for long-term evolutionary success in eukaryotes.

Acknowledgements. We thank Nicole El-Ali and Claire Hartmann for Nanopore sequencing, Jae Hur for the script used in generating tic plots, Janet Montgomery for overall editing, and Irina Arkhipova, Timothy Barraclough, Brian and Deborah Charlesworth, Antoine Hout, Paul Simion and Karine van Doninck for critical reading of the manuscript. This work was supported by Oxford Nanopore, the Harvard Faculty of Arts and Sciences and by an anonymous donor.

\section{References}

1. A. Weismann, On the significance of the polar globules. Nature 36, 607-609 (1887).

2. J. Maynard Smith, The Evolution of Sex (Cambridge University Press, 1978).

3. G. Bell, The Masterpiece of Nature: the evolution and genetics of sexuality (University of California Press, 1982).

4. N. H. Barton, B. Charlesworth, Why sex and recombination? Science. 281, 19861990 (1998).

5. S. P. Otto, The Evolutionary Enigma of Sex. Am. Nat. 174, S1-S14 (2009). 
6. I. Schon, K. Martens, P. van Dijk, Eds., Lost Sex : The evolutionary biology of parthenogenesis. (Springer, 2009).

7. D. B. Mark Welch, C. Ricci, M. Meselson, Lost Sex. Media, 259-279 (2009).

8. A. van Leewenhoeck, Observations, Communicated to the Publisher by Mr. Antony van Leewenhoeck, in a Dutch Letter of the 9th of Octob. 1676. Here English'd: concerning Little Animals by Him Observed in Rain-Well-Sea- and Snow Water; as Also in Water Wherein Pepper Had Lain In. Philos. Trans. R. Soc. London 12, 821-831 (1677).

9. A. van Leewenhoeck, Letter to the Royal Society December 25, 1702. Proc. R. Soc. London B 23, 1291-1311 (1702).

10. D. B. Mark Welch, J. L. Mark Welch, M. Meselson, Evidence for degenerate tetraploidy in bdelloid rotifers. Proc. Natl. Acad. Sci. 105, 5145-5149 (2008).

11. J. H. Hur, K. Van Doninck, M. L. Mandigo, M. Meselson, Degenerate tetraploidy was established before bdelloid rotifer families diverged. Mol. Biol. Evol. 26, 375383 (2009).

12. J.-F. Flot, et al., Genomic evidence for ameiotic evolution in the bdelloid rotifer Adineta vaga. Nature 500, 453-457 (2013).

13. R. W. Nowell, et al., Comparative genomics of bdelloid rotifers: Insights from desiccating and nondesiccating species. PLOS Biol. 16, e2004830 (2018).

14. W. S. Hsu, Oogenesis in the Bdelloidea rotifer Philodina roseola Ehrenberg. Cellule 57, 283-296 (1956).

15. W. S. Hsu, Oogenesis in Habrotrocha tridens (Milne). Biol. Bull. 111, 364-374 (1956).

16. N. D. Meadow, C. H. Barrows Jr., Studies on aging in a bdelloid rotifer .I. The effect of various culture systems and longevity and fecundity. J. Exp. Zool. 176, 303-314 (1971).

17. C. Ricci, Life histories of some species of Rotifera Bdelloidea. Hydrobiolgia 180, 175-180 (1983).

18. C. Ricci, U. Fascio, Life-history consequences of resource allocation of two bdelloid rotifer species. Hydrobiologia 299, 231-239 (1995).

19. M. Pagani, C. Ricci, A. M. Bolzern, Comparison of five strains of a 
parthenogenetic species, Macrotrachela quadricornifera (Rotifera, Bdelloidea) .II. Isoenzymatic patterns . Hydrobiologia 211, 157-163 (1991).

20. C. Ricci, Anhydrobiotic capabilities of bdelloid rotifers. Hydrobiologia 387/388, 321-326 (1998).

21. C. Ricci, M. Caprioli, Anhydrobiosis in bdelloid species, populations, and individuals. Integr. Comp. Biol. 45, 759-763 (2005).

22. C. Ricci, F. Perletti, Starve and survive: stress tolerance and life-history traits of a bdelloid rotifer. Funct. Ecol. 20, 340-346 (2006).

23. E. Gladyshev, M. Meselson, Extreme resistance of bdelloid rotifers to ionizing radiation. Proc. Natl. Acad. Sci. U. S. A. 105, 5139-5144 (2008).

24. A. Krisko, M. Leroy, M. Radman, M. Meselson, Extreme anti-oxidant protection against ionizing radiation in bdelloid rotifers. Proc. Natl. Acad. Sci. U. S. A. 109, 2354-2357 (2012).

25. M. B. França, a D. Panek, E. C. a Eleutherio, Oxidative stress and its effects during dehydration. Comp. Biochem. Physiol. A. Mol. Integr. Physiol. 146, 621631 (2007).

26. J. K. Fredrickson, et al., Protein oxidation: key to bacterial desiccation resistance? ISME J 2, 393-403 (2008).

27. C. G. Ehrenberg, Die Infusionsthierchen als vollkommene Organismen. EinBlick in das tiefere organische Laben der Natur (L. Voss, 1838).

28. C. T. Hudson, P. H. Gosse, The Rotifera or Wheel-Animalcules (Longmans, Green, 1886).

29. C. W. Birky, Positively negative evidence for asexuality. J. Hered. 101, 42-45 (2010).

30. C. Wesenberger-Lund, Contributions to the biology of the rotifera, part II: The periodicity and sexual periods. Kgl. Danske Vidensk. Selsk. Skr. Naturv. Og Math. Afd. 9. II, 1-230 (1930).

31. D. M. Ruderfer, S. C. Pratt, H. S. Seidel, L. Kruglyak, Population genomic analysis of outcrossing and recombination in yeast. Nat. Genet. 38, 1077-1081 (2006).

32. P. M. Magwene, et al., Outcrossing, mitotic recombination, and life-history trade- 
offs shape genome evolution in Saccharomyces cerevisiae. Proc. Natl. Acad. Sci. 108, 1987-1992 (2011).

33. D. B. Mark Welch, M. S. Meselson, Rates of nucleotide substitution in sexual and anciently asexual rotifers. Proc. Natl. Acad. Sci. 98, 6720-6724 (2001).

34. D. B. Mark Welch, M. Meselson, Evidence for the evolution of bdelloid rotifers without sexual reproduction or genetic exchange. Science $\mathbf{2 8 8 , 1 2 1 1 - 1 2 1 5}$ (2000).

35. A. R. Omilian, M. E. A. Cristescu, J. L. Dudycha, M. Lynch, Ameiotic recombination in asexual lineages of Daphnia. PNAS 103, 18638-18643 (2006).

36. S. Xu, A. R. Omilian, M. E. Cristescu, High rate of large-scale hemizygous deletions in asexually propagating Daphnia: Implications for the evolution of sex. Mol. Biol. Evol. 28, 335-342 (2011).

37. J. M. Flynn, F. J. J. Chain, D. J. Schoen, M. E. Cristescu, Spontaneous mutation accumulation in daphnia pulex in selection-free vs. competitive environments. Mol. Biol. Evol. 34, 160-173 (2017).

38. J. St. Charles, T. D. Petes, High-Resolution Mapping of Spontaneous Mitotic Recombination Hotspots on the 1.1 Mb Arm of Yeast Chromosome IV. PLoS Genet. 9, e1003434 (2013).

39. M. Hartfield, S. I. Wright, A. F. Agrawal, Coalescence and linkage disequilibrium in facultatively sexual diploids. Genetics 210, 683-701 (2018).

40. I. Schaefer, et al., No evidence for the "Meselson effect" in parthenogenetic oribatid mites (Oribatida, Acari). J. Evol. Biol. 19, 184-193 (2006).

41. D. A. Hickey, Selfish DNA: a sexually-transmitted nuclear parasite. Genetics 101, 519-531 (1982).

42. E. S. Dolgin, B. Charlesworth, The fate of transposable elements in asexual populations. Genetics 174, 817-827 (2006).

43. I. R. Arkhipova, M. Meselson, Transposable elements in sexual and ancient asexual taxa. Proc. Natl. Acad. Sci. 97, 14473-14477 (2000).

44. I. R. Arkhipova, M. Meselson, Diverse DNA transposons in rotifers of the class Bdelloidea. Proc. Natl. Acad. Sci. U. S. A. 102, 11781-11786 (2005).

45. I. Arkhipova, M. Meselson, Deleterious transposable elements and the extinction 
of asexuals. BioEssays 27, 76-85 (2005).

46. E. A. Gladyshev, M. Meselson, I. R. Arkhipova, A deep-branching clade of retrovirus-like retrotransposons in bdelloid rotifers. Gene 390, 136-145 (2007).

47. E. Gladyshev, I. Arkhipova, A subtelomeric non-LTR retrotransposon Hebe in the bdelloid rotifer Adineta vaga is subject to inactivation by deletions but not 5 ' truncations. Mob. DNA 1, 12 (2010).

48. F. Rodriguez, I. R. Arkhipova, Multitasking of the pirna silencing machinery: Targeting transposable elements and foreign genes in the Bdelloid Rotifer Adineta vaga. Genetics 203, 255-268 (2016).

49. J. L. Mark Welch, M. Meselson, Karyotypes of bdelloid rotifers from three families. Hydrobiologia 387/388, 403-407 (1998).

50. P. Simion, et al., Homologous chromosomes in asexual rotifer Adineta vaga suggest automixis. 10-12 (2020).

51. A. Signorovitch, J. Hur, E. Gladyshev, M. Meselson, Allele Sharing and Evidence for Sexuality in a Mitochondrial Clade of Bdelloid Rotifers. Genetics 200, 581-590 (2015).

52. K. E. Holsinger, N. C. Ellstrand, The Evolution and Ecology of Permanent Translocation Heterozygotes. Am. Nat. 124, 48-71 (1984).

53. A. Signorovitch, J. Hur, E. Gladyshev, M. Meselson, Evidence for meiotic sex in bdelloid rotifers. Curr. Biol. 26, R754-R755 (2016).

54. N. Debortoli, et al., Genetic Exchange among Bdelloid Rotifers Is More Likely Due to Horizontal Gene Transfer Than to Meiotic Sex. Curr. Biol. 26, 1-10 (2016).

55. C. G. Wilson, R. W. Nowell, T. G. Barraclough, Cross-Contamination Explains "Inter and Intraspecific Horizontal Genetic Transfers" between Asexual Bdelloid Rotifers. Curr. Biol. 28, 2436-2443.e15 (2018).

56. O. A. Vakhrusheva, et al., Recombination in a natural population of the bdelloid rotifer Adineta vaga. bioRxiv, 489393 (2018).

57. E. Lasek-Nesselquist, A Mitogenomic Re-Evaluation of the Bdelloid Phylogeny and Relationships among the Syndermata. PLoS One 7, e43554 (2012).

58. S. Xu, et al., High mutation rates in the mitochondrial genomes of Daphnia pulex. Mol. Biol. Evol. 29, 763-769 (2012). 
59. A. E. Tucker, M. S. Ackerman, B. D. Eads, S. Xu, M. Lynch, Population-genomic insights into the evolutionary origin and fate of obligately asexual Daphnia pulex. Proc. Natl. Acad. Sci. U. S. A. 110, 15740-15745 (2013).

60. A. Aguilera, et al., Distribution of Eukaryotes in an extremely acidic, high-metal river. Mol. Biol., 1-41 (2007).

61. R. Marotta, A. Uggetti, C. Ricci, F. Leasi, G. Melone, Surviving starvation: Changes accompanying starvation tolerance in a bdelloid rotifer. J. Morphol. 273, 1-7 (2012).

62. D. Fontaneto, T. G. Barraclough, K. Chen, C. Ricci, E. A. Herniou, Molecular evidence for broad-scale distributions in bdelloid rotifers: everything is not everywhere but most things are very widespread. Mol. Ecol. 17, 3136-3146 (2008).

63. C. G. Wilson, Desiccation-tolerance in bdelloid rotifers facilitates spatiotemporal escape from multiple species of parasitic fungi. Biol. J. Linn. Soc. 104, 564-574 (2011).

64. C. G. Wilson, P. W. Sherman, Spatial and temporal escape from fungal parasitism in natural communities of anciently asexual bdelloid rotifers. Proc. R. Soc. B Biol. Sci. 280, 20131255 (2013).

65. R. J. Ladle, R. A. Johnstone, O. P. Judson, Coevolutionary dynamics of sex in a metapopulation: escaping the Red Queen. Proc. R. Soc. Biol. Sci. Ser. B 253, 155-160 (1993).

66. L. T. Morran, O. G. Schmidt, I. A. Gelarden, R. C. Parrish, C. M. Lively, Running with the Red Queen: Host-parasite coevolution selects for biparental sex.

Science. 333, 216-218 (2011).

67. M. A. Brockhurst, et al., Running with the Red Queen: The role of biotic conflicts in evolution. Proc. R. Soc. B Biol. Sci. 281 (2014).

68. T. L. F. Leung, K. C. King, J. Wolinska, Escape from the Red Queen: An overlooked scenario in coevolutionary studies. Oikos 121, 641-645 (2012).

69. P. C. Phillips, Epistasis - The essential role of gene interactions in the structure and evolution of genetic systems. Nat. Rev. Genet. 9, 855-867 (2008).

70. M. Y. Hein, et al., A Human Interactome in Three Quantitative Dimensions 
Organized by Stoichiometries and Abundances. Cell 163, 712-723 (2015).

71. J. S. Hawe, F. J. Theis, M. Heinig, Inferring interaction networks from multi-omics data. Front. Genet. 10, 1-13 (2019).

72. O. Delaneau, et al., Chromatin three-dimensional interactions mediate genetic effects on gene expression. Science. 364, 452-462 (2019). 


\section{Figure Legends}

Fig. 1. The bdelloid M. quadricornifera, an unhatched egg shown at the same scale and a metaphase nucleus showing the 10 chromosomes characteristic of the species. The animal has attached itself to the glass slide by an adhesive secreted by its pedal gland, somewhat obscuring the tail.

Fig. 2. Histograms showing the distribution of divergence between the most similar homologs in each of the three pairs of isolates. (A) MA-CR, (B) MA-MM, (C) MM-CR. Alignments with identical or very nearly identical MA-MM sharing form a discrete class constituting half of the regions. Bin size $=0.05$ percent difference for the first bar in panel B. Otherwise 0.1 percent. Of the 333 alignments represented in the first bar of panel $B$, there are 315 in which MA and MM share identical homologs and 18 in which MM and MA share homologs that differ by no more than 15 substitutions or 0.09 percent.

Fig. 3. Histograms showing the distribution of heterozygosity in each of the three isolates. (A) CR, (B) MA. (C) MM. Bin size $=0.1$ percent difference.

Fig. 4. The mean frequency of identical MM-MA sharing in each of 8 bins of increasing length, each containing 76-79 intervals. Error bars represent standard errors.

\section{Table legends}

Table 1. Representative difference matrices, phylograms and tic plots for four alignments. Allele numbers in each alignment are arbitrary.

Table 2. Summary statistics. 
bioRxiv preprint doi: https://doi.org/10.1101/2020.08.06.239590; this version posted December 4, 2020. The copyright holder for this preprint (which was not certified by peer review) is the author/funder. All rights reserved. No reuse allowed without permission.

Fig. 1
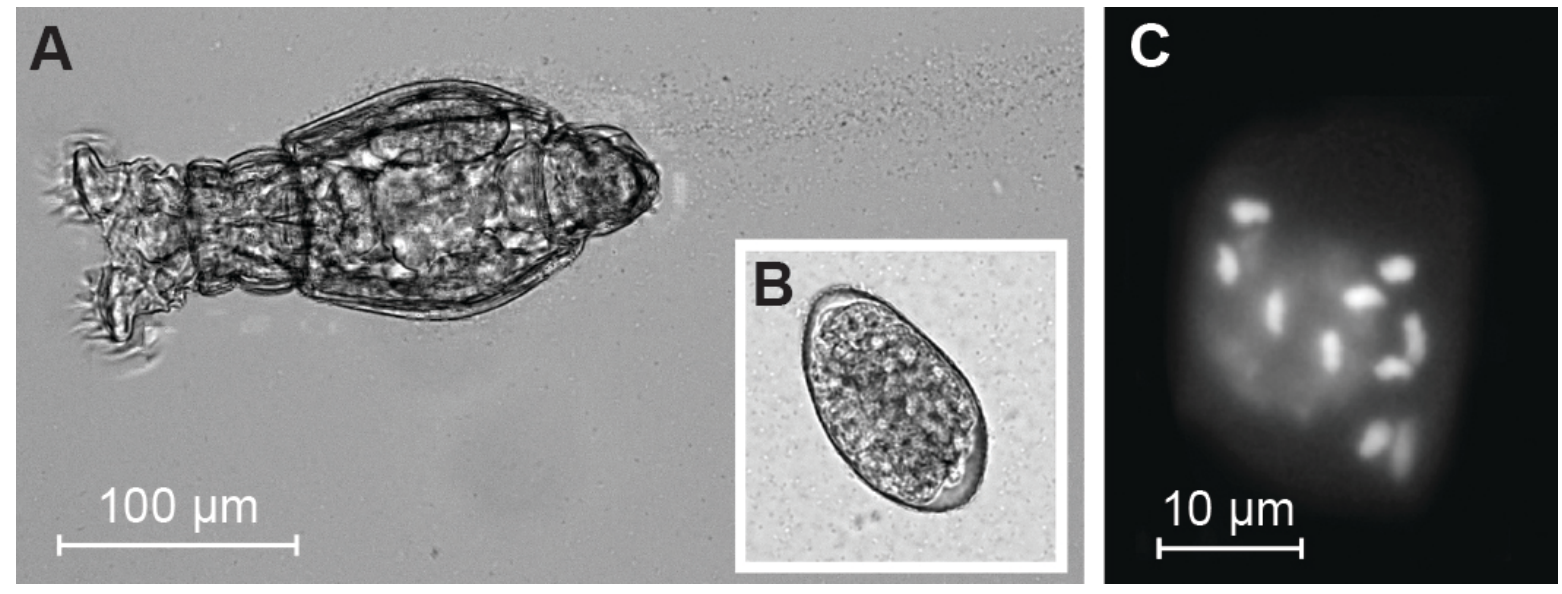
Fig. 2

A

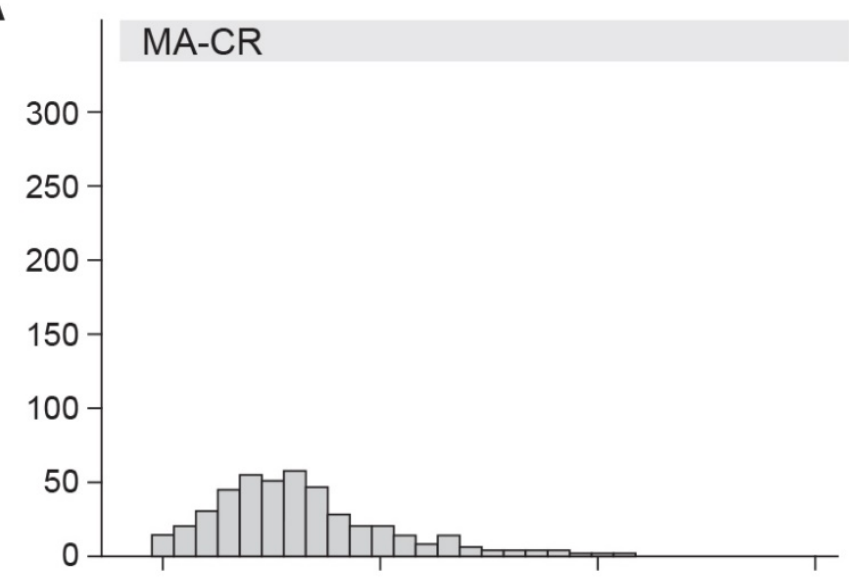

B

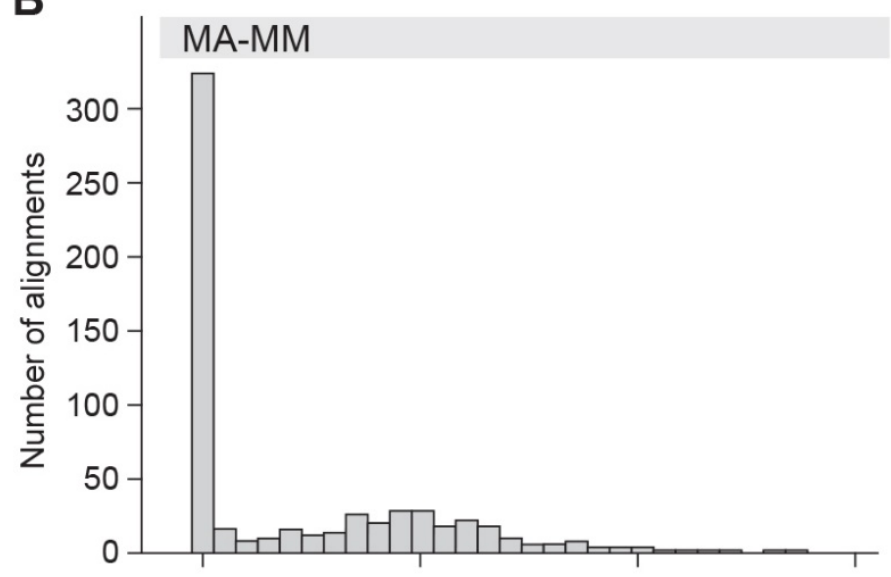

C

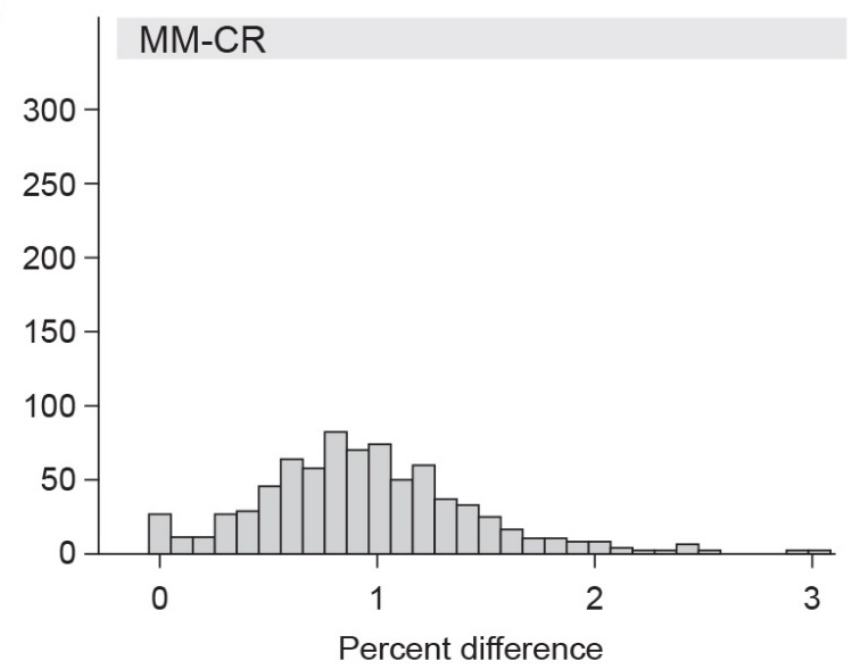


Fig. 3

A

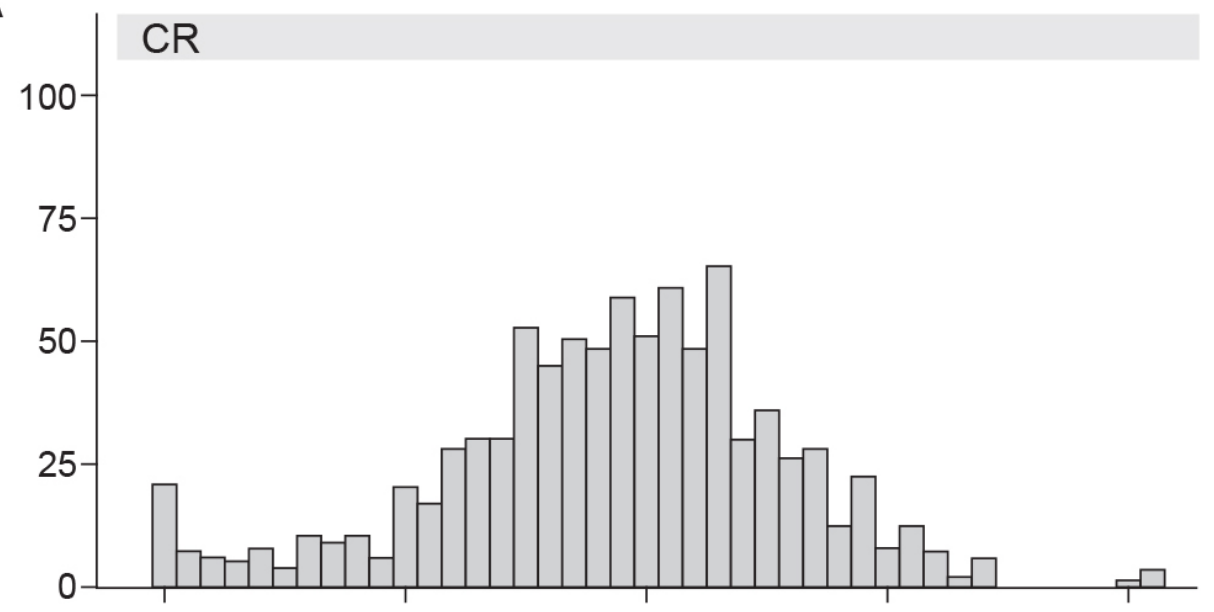

B

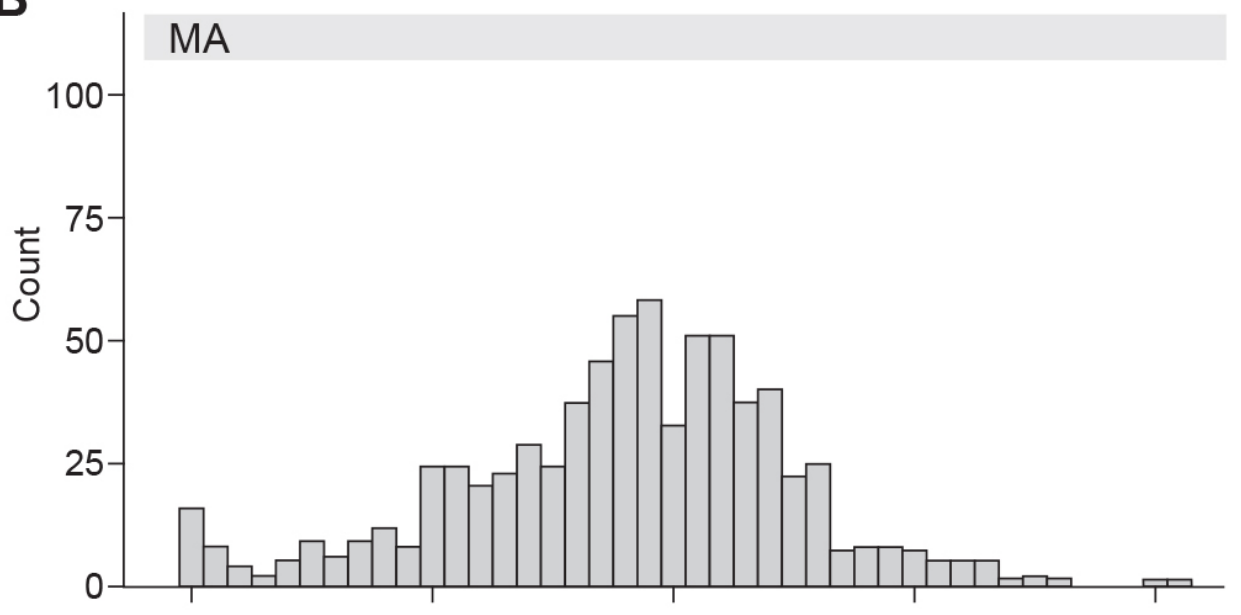

C

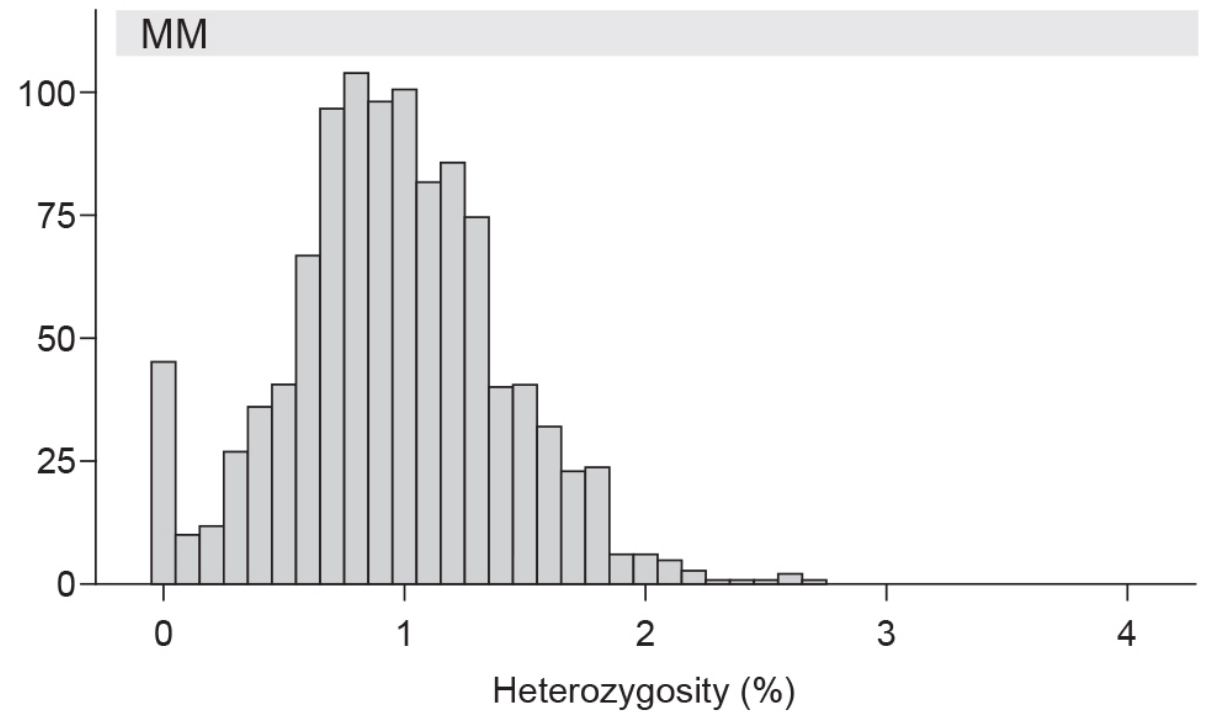


Fig. 4

\section{Length of Interval $(\mathrm{kb})$}

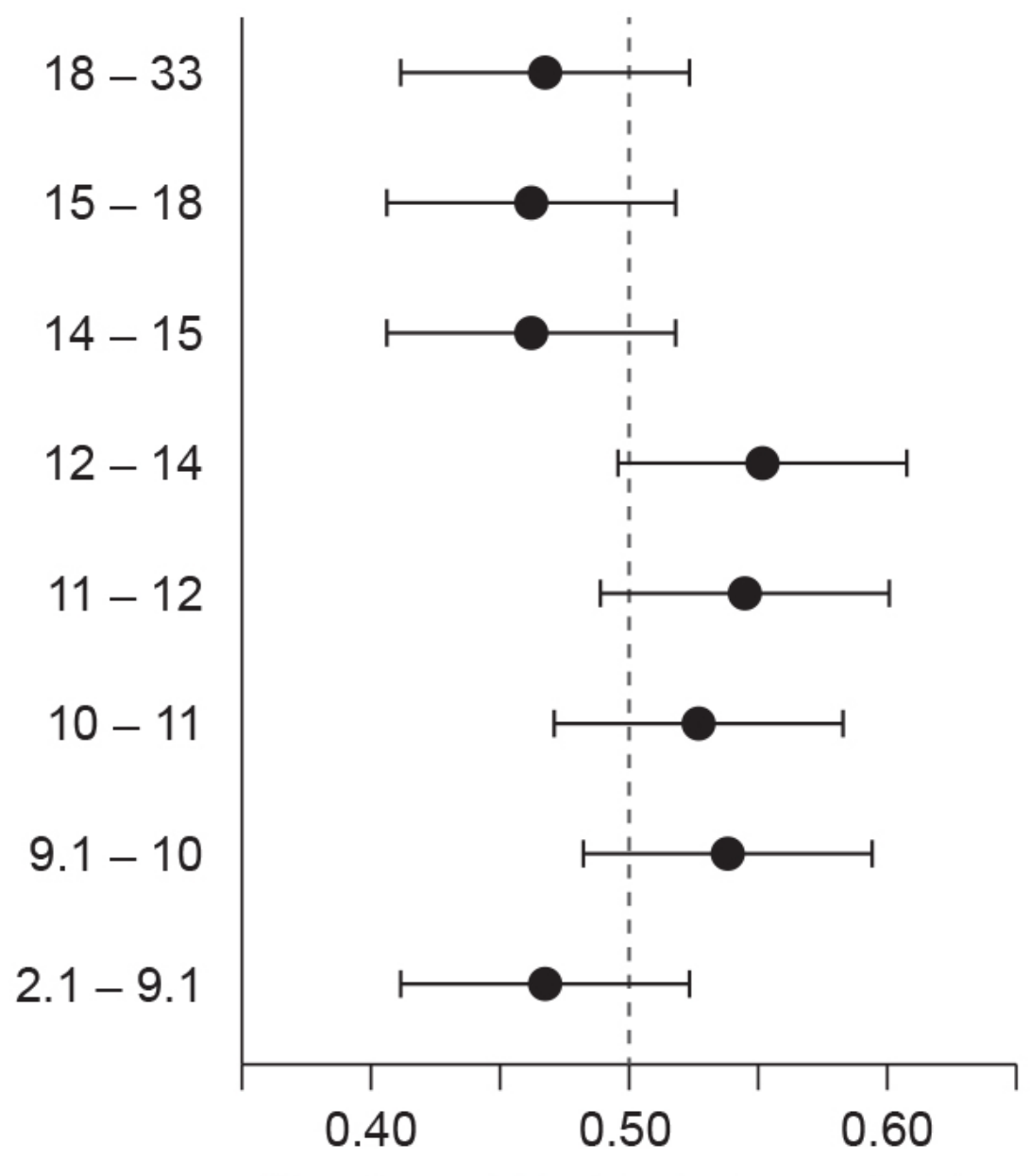

Fraction of identical sharers in bin 
Table 1

Number of differences

\section{\# $557 \quad 17,506 \mathrm{~kb} \quad$ No sharing}

\begin{tabular}{|c|c|c|c|c|}
\hline & MA1 & MA2 & MM1 & MM2 \\
\hline MA1 & - & 296 & 256 & 246 \\
MA2 & 1.7 & - & 413 & 394 \\
MM1 & 1.5 & 2.4 & - & 206 \\
MM2 & 1.4 & 2.3 & 1.2 & - \\
\hline
\end{tabular}

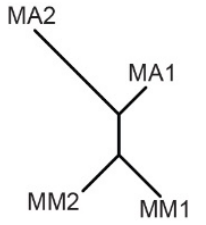

MA1-MA2

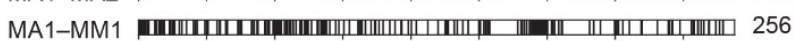
MA1-MM2

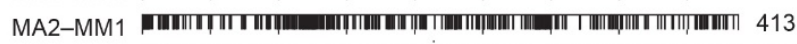

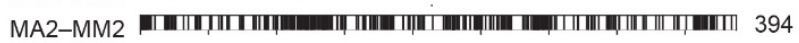
MM1-MM2

\section{\# $650 \quad 15,188 \mathrm{~kb} \quad$ MA - MM Sharing}

\begin{tabular}{|c|c|c|c|c|}
\hline & MA1 & MA2 & MM1 & MM2 \\
\hline MA1 & - & 241 & 0 & 134 \\
MA2 & 2.2 & - & 241 & 271 \\
MM1 & 2.7 & 1.6 & - & 134 \\
MM2 & 2.2 & 0 & 1.6 & - \\
\hline
\end{tabular}

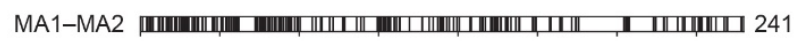
MA1-MM1 MA1-MM2 ாயாய 134 MA2-MM1 ||

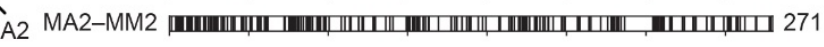

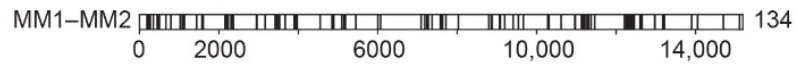

\section{\# $165 \quad 13,402 \mathrm{~kb} \quad$ MA - CR Sharing}

\begin{tabular}{|l|c|c|c|c|c|c|}
\hline & CR1 & CR2 & MA1 & MA2 & MM1 & MM2 \\
\hline CR1 & - & 311 & 155 & 311 & 312 & 307 \\
CR2 & 2.3 & - & 311 & 0 & 150 & 197 \\
MA1 & 1.1 & 2.3 & - & 311 & 307 & 322 \\
MA2 & 2.3 & 0 & 2.3 & - & 150 & 197 \\
MM1 & 2.3 & 1.1 & 2.3 & 1.1 & - & 190 \\
MM2 & 2.3 & 1.5 & 2.4 & 2.4 & 1.4 & - \\
\hline & MA2 \\
CR2 & MM2 \\
CR1
\end{tabular}

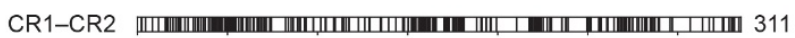

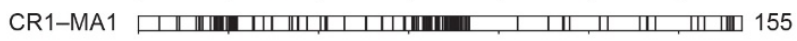

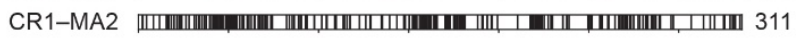

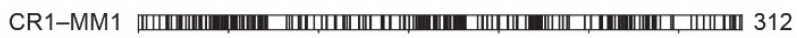

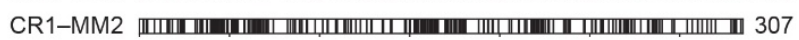
CR2-MA1 سயس CR2-MA2

CR2-MM1 $\square$ |

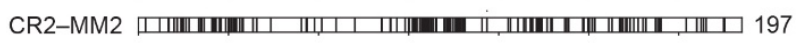

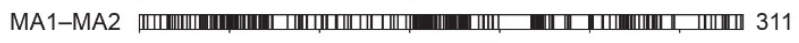
MA1-MM1 سயm

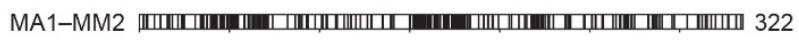

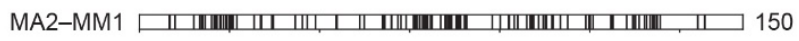

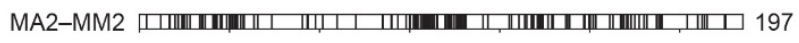

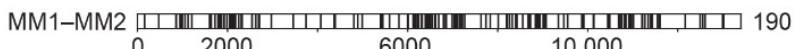

\section{\# $382 \quad 13,353$ kb $\quad$ MM - CR Sharing}

\begin{tabular}{|c|c|c|c|c|c|c|}
\hline & MA1 & MA2 & MM1 & MM2 & CR1 & CR2 \\
\hline MA1 & - & 236 & 109 & 143 & 109 & 248 \\
MA2 & 1.8 & - & 222 & 239 & 222 & 65 \\
MM1 & 0.8 & 1.7 & - & 119 & 0 & 233 \\
MM2 & 1.1 & 1.8 & 0.9 & - & 119 & 251 \\
CR1 & 0.8 & 1.7 & 0 & 0.9 & - & 233 \\
CR2 & 1.9 & 0.5 & 1.7 & 1.9 & 1.7 & - \\
\hline
\end{tabular}

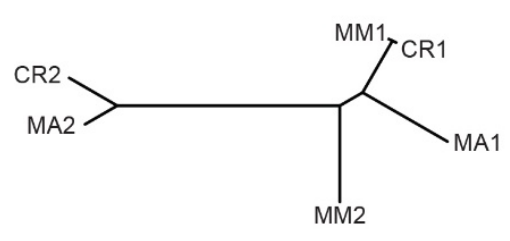

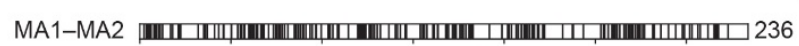

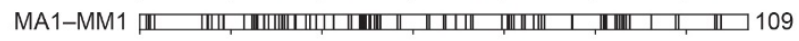
MA1-MM2 W 140

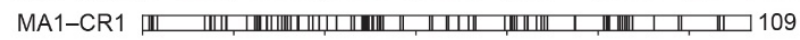
MA1-CR2 माए

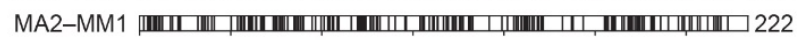
MA2-MM2 मा| MA2-CR1

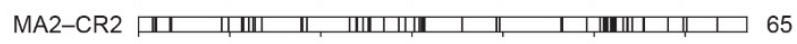
MM1-MM2 म 119 MM1-CR1

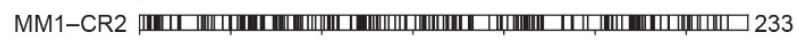

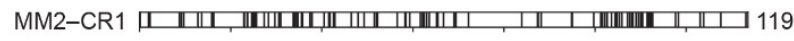

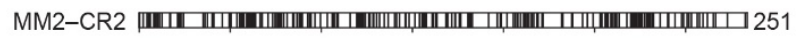

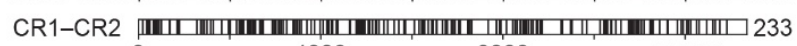
$\begin{array}{lll}4000 & 8000 & 12,000 \\ \text { Position in the alignment }\end{array}$ 
Table 2

\begin{tabular}{|c|c|c|c|c|c|c|c|c|c|c|}
\hline \multirow[b]{2}{*}{ Alignment } & \multirow[b]{2}{*}{$\begin{array}{l}\text { Size Range }(\mathrm{kb}) \\
\text { \# of Alignments }\end{array}$} & \multicolumn{3}{|c|}{$\begin{array}{l}\text { Identical Sharing } \\
\text { Number / Proportion }\end{array}$} & \multicolumn{3}{|c|}{$\begin{array}{c}\text { Homozygous } \\
\text { Number / Proportion }\end{array}$} & \multicolumn{3}{|c|}{ Average Heterozygosity } \\
\hline & & MA-MM & MA-CR & MM-CR & MA & MM & CR & MA & MM & CR \\
\hline \multirow{2}{*}{ MA-MM } & $2,149-32,937$ & 151 & & & 12 & 8 & & \multirow{2}{*}{0.019} & \multirow{2}{*}{0.010} & \\
\hline & 291 & 0.519 & & & 0.041 & 0.028 & & & & \\
\hline \multirow{2}{*}{ MA-CR } & $2,051-29,791$ & & 1 & & 1 & & 0 & \multirow{2}{*}{0.018} & & \multirow{2}{*}{0.018} \\
\hline & 110 & & 0.009 & & 0.009 & & 0 & & & \\
\hline \multirow{2}{*}{$\mathrm{MM}-\mathrm{CR}$} & $4,511-31,052$ & & & 7 & & 18 & 12 & & \multirow{2}{*}{0.010} & \multirow{2}{*}{0.020} \\
\hline & 445 & & & 0.016 & & 0.04 & 0.027 & & & \\
\hline \multirow{2}{*}{ MA-MM-CR } & $2,705-29,510$ & 164 & 11 & 11 & 1 & 4 & 3 & \multirow{2}{*}{0.018} & \multirow{2}{*}{0.009} & \multirow{2}{*}{0.018} \\
\hline & 331 & 0.495 & 0.033 & 0.033 & 0.003 & 0.012 & 0.009 & & & \\
\hline \multirow{2}{*}{ All alignments } & $2,051-32,937$ & 315 & 12 & 18 & 14 & 30 & 15 & \multirow{2}{*}{0.018} & \multirow{2}{*}{0.010} & \multirow{2}{*}{0.019} \\
\hline & 1,177 & 0.506 & 0.027 & 0.023 & 0.019 & 0.028 & 0.017 & & & \\
\hline
\end{tabular}

